\title{
PUMPCOM - um modelo para combinação de curvas e análise do desempenho de bombas
}

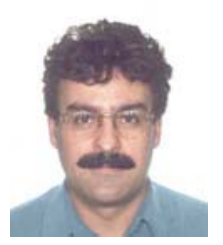

Camilo de L. T. de Andrade ${ }^{1}$, Richard G. Allen² \& Robin D. Wells ${ }^{3}$

\footnotetext{
1 Embrapa Milho e Sorgo, CP 151, CEP 35701-970, Sete Lagoas, MG. Fone: (31) 3779-1235. E-mail: camilo@cnpms.embrapa.br (Foto)

2 Universidade de Idaho, Research and Extension Center 3793N.3600E. Kimberly, Idaho, 83341, USA. Fone: (208) $423-6601$. E-mail: rallen@kimberly.uidaho.edu

${ }^{3}$ Consultor de Irrigação, Snake River Consulting, 3219 East, 3600 North, Kimberly, Idaho, 83341, USA
}

Protocolo $108-11 / 7 / 2001$

\begin{abstract}
Resumo: Um modelo de computador foi desenvolvido para combinar curvas de bombas em série e em paralelo e para determinar o desempenho da estação de bombeamento e das bombas que a compõem. Polinômios são ajustados aos dados das curvas de cada bomba e aos dados combinados da estação de bombeamento. A interpolação "spline" cúbica é utilizada para combinar curvas de bomba de capacidade diferente. Indicadores de desempenho da estação e de cada bomba, individualmente, são calculados para toda a faixa de vazão de operação do sistema. Uma interface gráfica permite ao usuário desenhar o croqui da estação de bombeamento e visualizar os dados de cada bomba e as equações ajustadas. Dados, gráficos e coeficientes de equações ajustadas podem ser impressos. Os cálculos do modelo foram verificados com planilha eletrônica.
\end{abstract}

Palavras-chave: curva de bomba, combinação de bombas, desempenho de bombas, modelo, Windows

\section{PUMPCOM - A model for pump curve combination and performance analysis}

\begin{abstract}
A computer model was developed to combine pump curves in series and in parallel and to determine the performance of the pump station and of individual pumps within the station. Polynomial equations are fited to individual pump curves and to the combined pump station curve. Cubic spline interpolation is employed to combine curves of pumps with different sizes. Performance indicators of the station and of each pump within the station are calculated for the entire station flow rate range. A graphical interface allows the user to draw the pump station layout and to view the discrete data entered along with the equations fit. Data entered, plots of the curves and equation coefficients can be printed out. PUMPCOM results were verified with spreadsheet calculations.
\end{abstract}

Key words: pump curve, pump combination, pump performance, model, Windows

\section{INTRODUÇÃO}

PUMPCOM é parte de um modelo maior, SPRINKMOD, desenvolvido para simular vazão e pressão em sistemas de irrigação pressurizados (Andrade, 1997; Andrade \& Allen, 1999; Andrade et al. 1999a; Andrade et al., 1999b). O modelo opera em ambiente Windows 95 e 98 e dispõe de interface gráfica para a entrada de dados e apresentação de resultados.

Qualquer sistema de distribuição de água ou de irrigação requer energia para mover a água da fonte para os pontos de distribuição. Na maioria dos casos, a energia requerida para mover a água é proveniente de um bomba hidráulica (Keller \& Bliesner, 1990). Bombas têm características de operação específicas, como curva de pressão, de eficiência, de potência e de NPSHr ("net positive suction head required") versus vazão
(Wheeler, 1993). Essas informações são geralmente fornecidas pelos fabricantes de bombas, através de catálogos.

Polinômios do segundo grau são comumente empregados para descrever matematicamente curvas características de bombas (Gessler, 1981; Tullis, 1989), embora polinômios de outros graus tenham sido experimentados (Ramalan, 1988; Molina, 1991; Wheeler, 1993). Polinômios de grau acima de dois devem ser empregados com cautela, pois valores absurdos podem ser obtidos entre dois pontos, sobretudo quando poucos pontos são empregados no ajuste do modelo (Mathews, 1987). Uma forma alternativa para descrever curvas de bombas é a "spline" cúbica, aplicada com sucesso por Molina (1991) para encontrar o ponto de operação de um sistema de irrigação. Wheeler (1993) sugere que "spline" cúbica seja empregada no lugar de polinômios de quarto grau. A teoria sobre "spline" cúbica é descrita em detalhes por Manson (1987) e Kincaid \& Cheney (1991). 
Sistemas de distribuição urbanos e de irrigação, normalmente operam numa faixa ampla de vazões, devido a mudanças na demanda, variações no nível de reservatórios ou nas posições das laterais ou, simplesmente, devido a mudanças nas perdas de carga decorrentes da operação de válvulas ou do processo de envelhecimento das tubulações. Nessas situações, é desejável a utilização de mais de uma bomba combinada em série e/ou em paralelo.

Bombas em série são empregadas quando uma curva característica muito inclinada é desejável (alto valor de dH/dQ), tal como quando a vazão deve ser similar para mudanças bruscas na pressão. Essa situação pode ocorrer quando laterais de irrigação são mudadas de posições elevadas no campo para posições mais baixas e vice-versa e se deseja manter a vazão nas mesmas (Allen, 1992). Além do mais, bombas em série são utilizadas quando uma única bomba não consegue suprir a pressão necessária a um sistema hidráulico. Para bombas operando em série, cada uma aplica energia adicional a uma mesma vazão que passa por elas. A pressão final (combinada) é essencialmente a soma das pressões individuais de cada bomba, menos a perda de carga ao longo do caminho da água (Tullis, 1989; Keller \& Bliesner, 1990 e Krivchenko, 1994).

Bombas em paralelo são utilizadas sempre que uma curva característica plana (pequeno valor de $\mathrm{dH} / \mathrm{dQ}$ ) é requerida, ou seja, a pressão se reduz suavemente com o aumento da vazão (Allen, 1992). Uma situação dessas ocorre quando a demanda de água varia com tempo, como durante um dia em uma cidade ou como ocorre ao longo do ciclo das culturas numa área irrigada ou, ainda, quando laterais são ligadas ou desligadas em um sistema de irrigação. Bombas são também conectadas em paralelo por questões de segurança para o sistema hidráulico ou para simplificar a manutenção. Em cidades, é comum a utilização de duas ou três bombas em paralelo, cada uma com capacidade para suprir $50 \%$ ou $33 \%$ da vazão requerida (Tullis, 1989). Quando bombas operam em paralelo, elas trabalham com uma pressão a jusante comum. As curvas combinadas são geradas de forma similar à das bombas em série, exceto que vazões em vez de pressões, são somadas para uma pressão comum (Tullis, 1989; Keller \& Bliesner, 1990, Krivchenko, 1994).

O NPSHr combinado para bombas em paralelo é aquele mais limitante entre as bombas, o maior NPSHr neste caso, enquanto para bombas em série o NPSHr combinado é igual ao da bomba que estiver a montante, pois as demais estarão operando com pressões positivas na sucção.

Quando bombas são combinadas em série ou em paralelo, é importante analisar a pressão, a eficiência, o NPSHr e a potência combinada e de cada bomba, individualmente, para toda a faixa de vazões na qual o sistema hidráulico irá operar. É desejável ter o ponto de operação das bombas o mais próximo possível do ponto de melhor eficiência, tanto para bombas combinadas quanto para cada uma, individualmente. Combinações desiguais podem reduzir a eficiência, causar retorno de água por alguma bomba ou fazer alguma bomba operar como turbina. Nessas circunstâncias, há desperdício de energia e o motor acoplado às bombas pode ser danificado. A combinação de curvas de bomba manualmente, é trabalhosa, fazendo com que programas de computador e planilhas eletrônicas sejam empregados. Ormsbee \& Walski (1989) desenvolveram um sistema gráfico para a combinação de bombas em série e/ou em paralelo, o qual foi empregado para a otimização do bombeamento de água de sistemas de distribuição de água de cidades. O modelo não é prático para a combinação de mais de duas bombas. Tarquin \& Dowdy (1989) desenvolveram um modelo para avaliar a eficiência e o custo de bombeamento de uma estação de bombeamento específica. Analisando-se a eficiência e a potência consumida por bombas combinadas de diversas maneiras, a melhor combinação poderia ser selecionada. Wells \& Busch (1981) desenvolveram um procedimento não gráfico para selecionar a combinação mais apropriada de bombas para satisfazer certa demanda de pressão e vazão e demonstraram o modelo com nove bombas operando em paralelo.

Considerando-se os poucos modelos existentes para a combinação e análise de curvas de bombas, desenvolveu-se este modelo com interface amigável, capaz de combinar bombas em série e/ou em paralelo, de tal forma que a potência, NPSHr, vazão e pressão, pudessem ser analisadas e visualizadas, mesmo em estações de bombeamento complexas.

\section{DESCRIÇÃO E DESENVOLVIMENTO DO MODELO}

Para que modelos possam simular sistemas hidráulicos que contenham bombas, a sua curva característica de pressão-vazão deve ser conhecida. PUMPCOM permite que o usuário ajuste polinômios de até quinto grau aos dados discretos originais de curvas de bombas fornecidos em catálogos pelos fabricantes; todavia, os polinômios são empregados apenas para testar os dados e eliminar discrepâncias. $\mathrm{O}$ modelo permite que o desempenho da estação de bombeamento e de cada uma das bombas que a compõem possa ser avaliado para toda a faixa de vazão de operação do sistema hidráulico. A lógica empregada no desenvolvimento do modelo e as suas peculiaridades, serão discutidas na medida em que o mesmo for sendo descrito.

\section{Unidades}

Dois sistemas de unidade podem ser utilizados no PUMPCOM: o sistema métrico e o sistema inglês. No primeiro, vazão, pressão, diâmetro de tubulação e eficiência são representados respectivamente, em litros por segundo, metro, milímetro e porcentagem; para o sistema inglês, as unidades são, respectivamente, galões por minuto, pé, polegada e porcentagem. O usuário pode escolher entre trabalhar com um ou com outro sistema ou converter os arquivos de um sistema para outro. Internamente, todos os dados são convertidos para um sistema comum, antes de se iniciar os cálculos para a simulação.

\section{Arquivos de curva característica de bomba}

$\mathrm{O}$ usuário tem que criar um arquivo para cada curva de bomba que se pretende combinar, mesmo que a estação de bombeamento tenha apenas uma bomba. $\mathrm{O}$ mesmo arquivo pode ser empregado depois em outras estações de bombeamento e em outros sistemas de irrigação, sem a necessidade de reentrar com os dados.

Uma caixa de diálogo é empregada para entrar e editar dados de pressão, vazão, eficiência e NPSHr, para uma curva de bomba (Figura 1). Um mínimo de seis e um máximo de 21 pontos da curva de bomba devem ser fornecidos para que um polinômio de até quinto grau possa ser ajustado. PUMPCOM é dotado de 
uma rotina capaz de detectar qualquer tipo de erro na entrada de dados e avisar o usuário para corrigí-los.

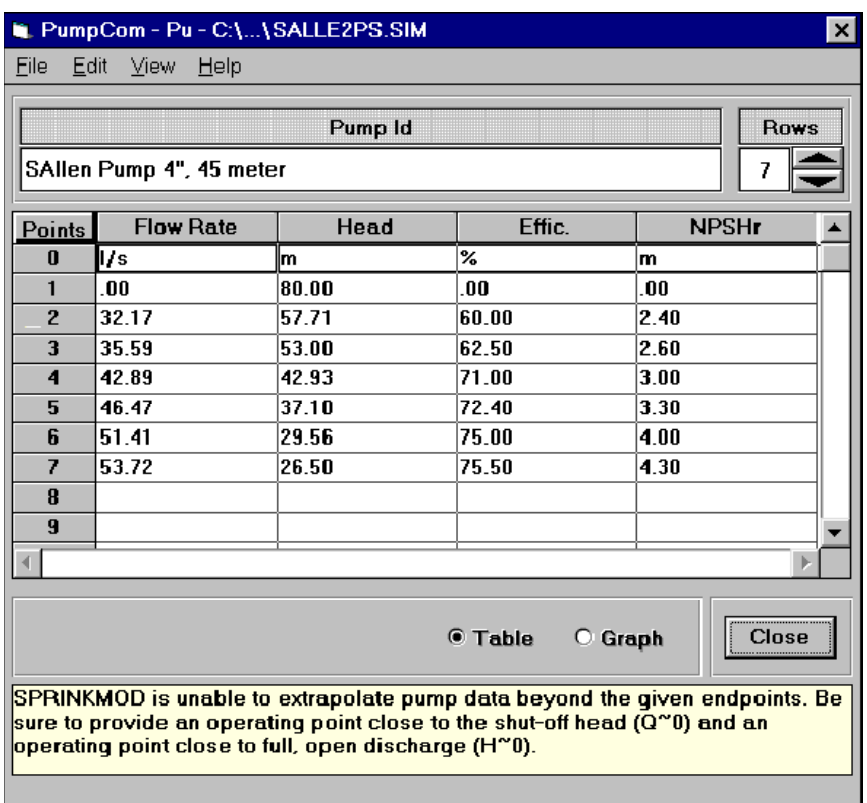

Figura 1. Caixa de diálogo para entrada e edição de dados de curva de bomba

Uma outra rotina verifica a consistência dos dados de vazão versus pressão, para assegurar que a pressão decresça monotonicamente com o aumento da vazão. Esta é uma exigência para modelagem de bombas, que faz com que, para cada valor de pressão, só exista um valor de vazão. O usuário pode modificar o grau do polinômio e analisar o efeito no gráfico e na tabela de erro padrão da regressão (Figura 2). Procedimentos convencionais para regressão são empregados para ajustar os polinômios (Mathews, 1987; Kincaid \& Cheney, 1991). O usuário pode gravar o arquivo, visualizar o gráfico, imprimir dados e gráfico e editar outros arquivos.

\section{Arquivos de estação de bombeamento}

A estação de bombeamento pode ter uma ou mais bombas com diferentes capacidades e características acopladas entre

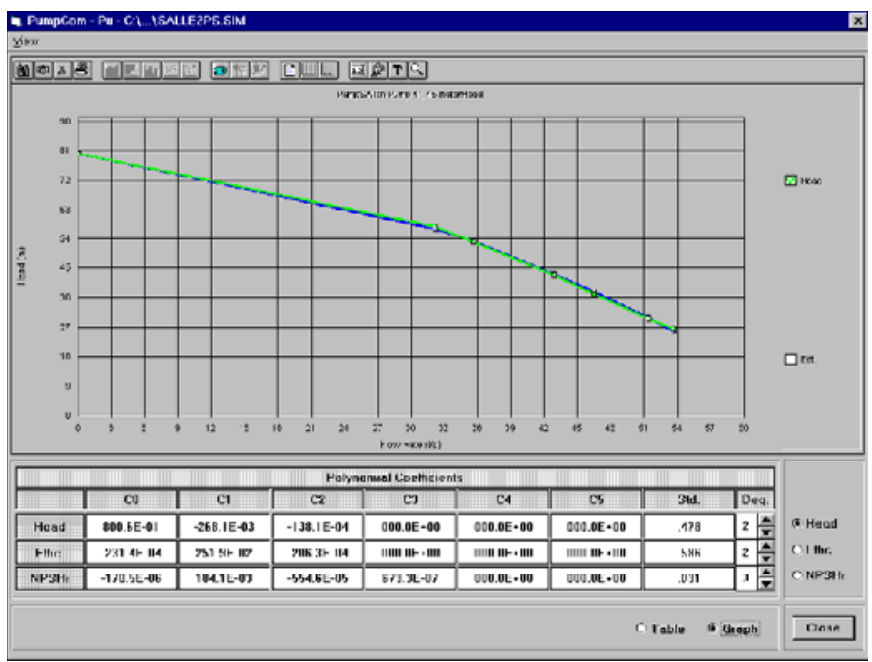

Figura 2. Dados de pressão-vazão e coefícientes dos polinômios ajustados si. Bombas na estação de bombeamento podem estar conectadas em série ou em paralelo ou em ambos. O programa é capaz de acompanhar intricados leiautes e gerar uma única curva combinada. A localização das bombas dentro da estação é descrita graficamente, empregando-se uma grade e ícones com os quais o usuário pode desenhar o leiaute, incluindo tubulações e conexões. Os passos para a criação do leiaute de uma estação de bombeamento são descritos a seguir.

\section{Seleção de arquivos de bombas para serem combinadas}

Arquivos de bombas a serem utilizadas numa estação de bombeamento podem ser selecionados a partir de uma lista de arquivos disponíveis (Figura 3). O usuário pode navegar por diferentes diretórios no disco, para encontrar os arquivos das bombas que deseja combinar. Qualquer número de arquivos pode ser selecionado e em qualquer ordem. Quando um arquivo de estação de bombeamento é editado, PUMPCOM procura pelos arquivos individuais de curvas de bombas cujos nomes e seqüência de diretórios ("path") estão armazenados no arquivo da estação de bombeamento e os seleciona e, se por alguma razão algum arquivo não é encontrado, o seu nome é indicado ao usuário, que deve anotá-lo e tentar encontrá-lo em algum outro diretório ou disco; se um arquivo individual de curva de bomba não for encontrado, o arquivo da estação de bombeamento que o contém não pode mais ser editado e o processo de edição tem que ser abortado; todavia, o arquivo da estação de bombeamento existente pode ser analisado e empregado pelo SPRIKMOD.

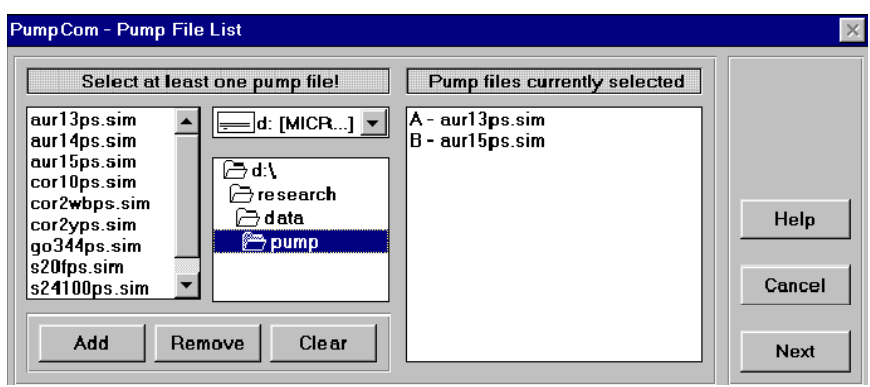

Figura 3. Caixa de diálogo para seleção de arquivos individuais de bombas, mostrando dois arquivos selecionados

\section{Construção do leiaute da estação de bombeamento}

PUMPCOM foi projetado para ter uma interface amigável, para ser capaz de combinar bombas de qualquer capacidade em série e/ou em paralelo e para permitir a inclusão de perdas de carga localizada nos cálculos da curva combinada.

Após a seleção dos arquivos individuais de bombas a serem combinadas (Figura 3), uma nova janela é mostrada, na qual o usuário pode desenhar o leiaute da estação de bombeamento em uma grade (Figura 4). A lista das bombas previamente selecionadas é armazenada numa caixa tipo lista ("dropdown box"). Uma bomba dessa caixa pode ser selecionada e colocada em qualquer local da grade. Um outra caixa contém ícones de conexões que podem ser colocadas entre as bombas, para conectá-las, de forma que um sistema hidráulico possa ser montado. Com esse procedimento, o leiaute de uma estação de bombeamento pode ser desenhado o mais próximo possível da realidade. Algumas restrições existem, todavia: (i) o fluxo de água deve ir da esquerda para a direita na grade; (ii) bombas 
não podem ser colocadas na coluna da extrema direita e conexões não podem ser colocadas na coluna da extrema esquerda; (iii) somente uma saída pode existir e deve ser um tubo reto colocado na coluna da extrema direita; (iv) exceto pela saída, conexões devem estar conectadas umas às outras ou às bombas; (v) bombas podem ter o seu lado esquerdo livre, o que indica que é a sua sucção, ligada diretamente à fonte de água; (vi) não é permitida a presença de circuito fechado ("loops").

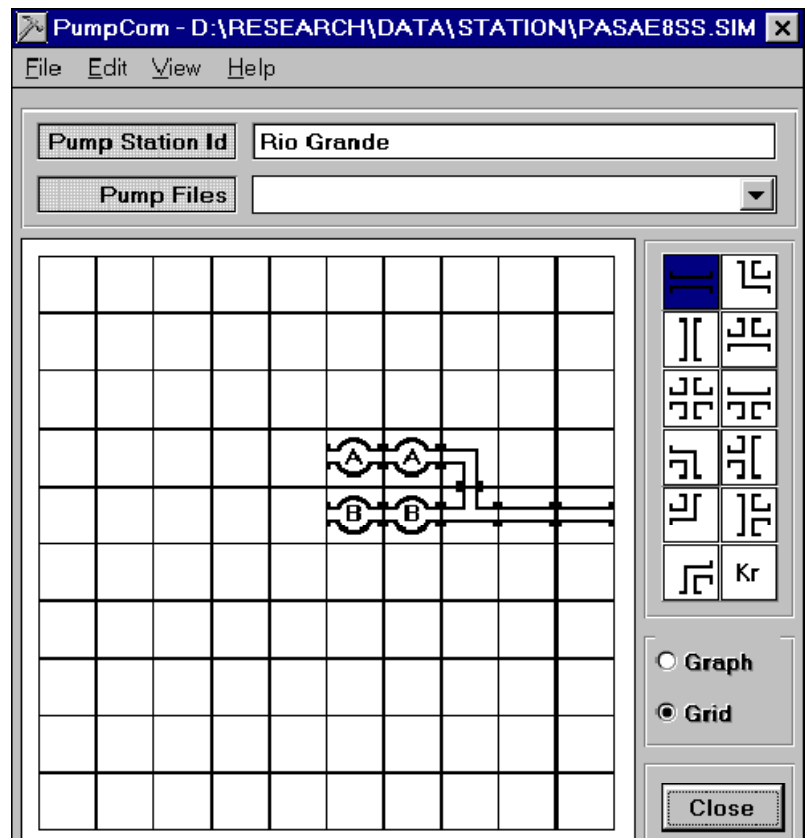

Figura 4. Leiaute de uma estação de bombeamento indicando a grade, duas bombas tipo A em série, duas bombas diferentes tipo B em série e os dois grupos conectados em paralelo

\section{Perdas de carga localizada}

Coeficientes de perda de carga localizada, $\mathrm{K}_{\mathrm{r}}$, podem ser associados a cada bomba; assim, perdas de carga causadas por conexões a jusante da bomba podem ser computadas e subtraídas da pressão gerada por ela. Da mesma forma, coeficientes de perda de carga localizada podem ser associados a cada uma das intersecções (bifurcações ou "trifurcações"), permitindo que a pressão a jusante delas seja corrigida. A perda de carga localizada de qualquer secção de uma estação de bombeamento para a qual foi especificado um valor de $\mathrm{K}_{\mathrm{r}}$ e um diâmetro é computada pela seguinte equação (Flammer et al., 1986; Keller \& Bliesner, 1990):

$$
\mathrm{h}_{1}=\mathrm{K}_{\mathrm{r}} \frac{\mathrm{V}^{2}}{2 \mathrm{~g}}
$$

em que:

$\mathrm{h}_{1} \quad$ - perda de carga localizada, $\mathrm{L}$

$\mathrm{K}_{\mathrm{r}}$ - coeficiente de perda de carga localizada, adimensional

$\mathrm{V}$ - velocidade média, $\mathrm{LT}^{-1}$

g - aceleração devido a gravidade, $\mathrm{LT}^{-2}$

$\mathrm{O}$ valor de $\mathrm{K}_{\mathrm{r}}$ a ser fornecido deve contemplar o efeito de todas as peças que estiverem a jusante até uma nova bomba ou intersecção e pode incluir curvas, expansões, contrações, válvulas, registros e grades ou telas. Geralmente, perdas de carga localizada dentro das bombas já são computadas pelo fabricante e incluídas na curva de pressão-vazão dos catálogos.

\section{Escaneamento do leiaute da estação de bombeamento}

O processo de combinação das curvas é disparado quando o usuário pede para ver o gráfico da curva combinada ou para ver os dados de desempenho de uma bomba no leiaute, dando um clique com o botão direito do mouse no ícone da bomba. Como primeiro passo, PUMPCOM escaneia a grade e verifica as ligações entre bombas e entre conexões. Acoplamentos errados são indicados ao usuário; em seguida, começando pela saída, o leiaute é navegado e a seqüência na qual as bombas serão combinadas é armazenada em matrizes de dados. Índices e sinalizadores ("flags") são empregados para organizar seqüências de bombas, interseções e ramificações, numa forma hierárquica. Sinalizadores indicam se uma seqüência de bombas armazenadas na matriz com o mesmo índice deve ser combinada em série ou em paralelo. Após o escanemento do leiaute, PUMPCOM dirige suas ações para um módulo de cálculo onde as combinações propriamente ditas são realizadas. $\mathrm{O}$ emprego desse procedimento reduz a demanda por memória do computador, por não utilizar recursos gráficos durante os cálculos. Códigos-fonte mais simples, menores e mais fácil de se manter são empregados; além do mais, a mesma matriz contendo dados da estação de bombeamento é empregada mais tarde para gerar dados de desempenho para cada uma das bombas.

\section{Combinação das curvas de bomba}

A matriz com os dados da estação de bombeamento é lida, começando-se pelo menor índice, que é aquele referente à bomba que está na posição mais a montante no leiaute. Para cada bomba encontrada, o respectivo arquivo de dados é aberto e lido e as informações armazenadas em matrizes temporárias, organizadas de acordo com os índices das interseções e ramificações. A faixa de vazões possível de cada bomba (de acordo com seus dados originais) é dividida em 20 intervalos regulares, para gerar uma série de dados discretos. O limite inferior dessa série é zero e o limite superior é o maior valor de vazão fornecido pelo usuário, quando da criação do arquivo individual da bomba. Para cada valor de vazão, valores de pressão, eficiência e NPSHr são gerados, empregando-se o polinômio ajustado pelo usuário.

\section{Bombas em série}

Quando um sinalizador para bombas em série é encontrado, todas as curvas com o mesmo índice de interseção e de ramificação são combinadas, somando-se as pressões para valores iguais de vazão (Tullis, 1989). Se existe perda de carga localizada entre as bombas, a Eq. 1 é empregada para estimá-la e o valor da perda é subtraído da pressão da bomba que estiver a montante, antes de se somar com a pressão da bomba que estiver a jusante. Se os valores discretos de vazão de duas bombas em série não são iguais, como no caso de bombas com diferentes capacidades sendo combinadas, PUMPCOM gera uma nova série de 21 dados discretos de vazão, começando com zero até o menor valor de vazão máxima entre todas as 
bombas que estão sendo combinadas em série. A pressão para cada valor discreto de vazão é determinada empregando-se "spline" cúbica (Kincaid \& Cheney, 1991) para interpolar da tabela de vazão-pressão.

Utilizando-se a equação para eficiência combinada para bombas em série, como descrito em Keller \& Bliesner (1990), e a equação para potência no eixo da bomba (Tullis, 1989) e assumindo-se que não há perda de carga entre as bombas, a eficiência combinada é estimada por:

$$
\mathrm{E}_{\mathrm{ps}}=\frac{\sum_{\mathrm{i}=1}^{\mathrm{i}=\mathrm{n}} \mathrm{h}_{\mathrm{p}_{\mathrm{i}}}}{\sum_{\mathrm{i}=1}^{\mathrm{i}=\mathrm{n}}\left(\frac{\mathrm{h}_{\mathrm{p}_{\mathrm{i}}}}{\mathrm{E}_{\mathrm{p}_{\mathrm{i}}}}\right)}
$$

em que:

$$
\begin{array}{ll}
\mathrm{E}_{\mathrm{ps}} & \text { - eficiência combinada para bombas em série, \% } \\
\mathrm{E}_{\mathrm{pi}} & \text { - eficiência da bomba i, \% } \\
\mathrm{h}_{\mathrm{pi}} & \text { - pressão da bomba i, L } \\
\mathrm{n} & \text { - número de bombas em série, adimensional }
\end{array}
$$

\section{Bombas em paralelo}

Sinalizadores para bombas em paralelo são colocados pelo PUMPCOM quando há uma interseção que recebe fluxo de água de duas ou três ramificações e o direciona para um tubo ou bomba que esteja a jusante. No processo de leitura da matriz da estação de bombeamento, quando um sinalizador para bombas em paralelo é encontrado, os dados armazenados nela com o mesmo índice, são preparados para a combinação. Esses dados podem ser de uma ou mais bombas em série ou da combinação de bombas em paralelo ocorridas a montante, os quais, para o PUMPCOM, funcionam como uma bomba fictícia. Um conjunto de 21 dados discretos de pressão é gerado, iniciando com o maior valor mínimo e terminando como o maior valor máximo de pressão entre as bombas a serem combinadas em paralelo. "Spline" cúbica é utilizada para interpolar e determinar valores de vazão correspondentes a cada um dos valores de pressão. Valores de vazão para um mesmo valor de pressão são somados para gerar a tabela final de dados de pressão-vazão para as bombas combinadas em paralelo. As perdas de carga localizada no trecho da interseção até a próxima bomba ou interseção a jusante são calculadas para cada valor de vazão e subtraídas dos correspondentes valores combinados de pressão. Tal como foi feito para bombas em série, a eficiência combinada é uma média ponderada das eficiências das bombas em paralelo, utilizando-se a vazão como peso. A expressão derivada para determinar a eficiência combinada para bombas em paralelo é:

$$
\mathrm{E}_{\mathrm{pp}}=\frac{\sum_{\mathrm{i}=1}^{\mathrm{i}=\mathrm{n}} \mathrm{Q}_{\mathrm{i}}}{\sum_{\mathrm{i}=1}^{\mathrm{i}=\mathrm{n}}\left(\frac{\mathrm{Q}_{\mathrm{i}}}{\mathrm{E}_{\mathrm{p}_{\mathrm{i}}}}\right)}
$$

sendo:

$$
\mathrm{E}_{\mathrm{pp}} \quad \text { - eficiência combinada para bombas em paralelo, \% }
$$

$\mathrm{E}_{\mathrm{pi}} \quad$ - eficiência para bomba i, \%

$\mathrm{Q}_{\mathrm{i}} \quad$ - vazão para bomba $\mathrm{i}, \mathrm{L}^{3} \mathrm{~T}^{-1}$

n - número de bombas em paralelo, adimensional

Depois que todas as bombas de uma ramificação ou conectadas a uma interseção são combinadas em série ou em paralelo, os dados de pressão, vazão e eficiência são armazenados em matrizes temporárias com os índices apropriados. Esse procedimento é repetido até que toda a matriz da estação de bombeamento seja lida e o sinalizador indicando a saída seja encontrado. Nesse ponto, a curva característica combinada está então calculada e os dados são armazenados numa matriz.

O NPSHr para a estação de bombeamento é determinado selecionando-se o maior valor entre os NPSHrs de todas as bombas cuja sucção esteja livre, ou seja, não esteja ligada a outra bomba ou conexão.

\section{Desempenho da estação de bombeamento}

PUMPCOM vai além do processo de simplesmente gerar curvas de bomba combinadas. Para cada ponto da curva combinada e para cada uma das bombas que fazem parte da estação de bombeamento, o modelo computa e armazena dados de pressão a montante e a jusante e eficiência.

A matriz da estação de bombeamento é lida de trás para frente, começando na saída e caminhando na direção a montante. Um valor de vazão diferente de zero e o seu correspondente valor de pressão são selecionados da tabela de vazão-pressão da estação de bombeamento. A perda de carga localizada é computada e adicionada ao valor de pressão, já que se está caminhando na direção contrária ao fluxo de água. Quando um sinalizador que representa uma interseção é encontrado, um procedimento iterativo é empregado para se calcular o par vazão-pressão da ramificação a jusante que produz o valor da pressão na interseção. Quando um sinalizador para bomba é encontrado, "spline" cúbica é utilizada para interpolar a tabela de vazão-pressão daquela bomba e encontrar a vazão que produz o valor da pressão a sua jusante. Todos os valores de pressão e vazão calculados para cada segmento do leiaute são armazenados em matrizes com os índices apropriados para interseção e ramificação. Esse processo continua até que seja determinada a vazão individual das bombas da estação, para qual existe uma correspondente vazão combinada. Todas as pressões a montante e a jusante de cada bomba são calculadas e armazenadas. A eficiência e o NPSHr de cada bomba e para cada um dos valores discretos de vazão são determinados empregando-se as respectivas equações polinomiais ajustadas quando da criação dos arquivos individuais das bombas.

Pressão, eficiência, NPSHr e vazão para toda a faixa de operação da estação de bombeamento e para cada uma das bombas que a compõem são armazenados em matrizes. Quando o usuário dá um clique com o botão direito do mouse no ícone de uma bomba na grade do leiaute, os dados para a estação e para aquela bomba são mostrados (Figura 5).

Esta é uma das mais importantes características do modelo, pois permite que o usuário analise o desempenho da estação de bombeamento e de cada uma de suas bombas para cada ponto da curva combinada, ou seja, para uma faixa grande de possíveis pontos de operação. Problemas de baixo desempenho 


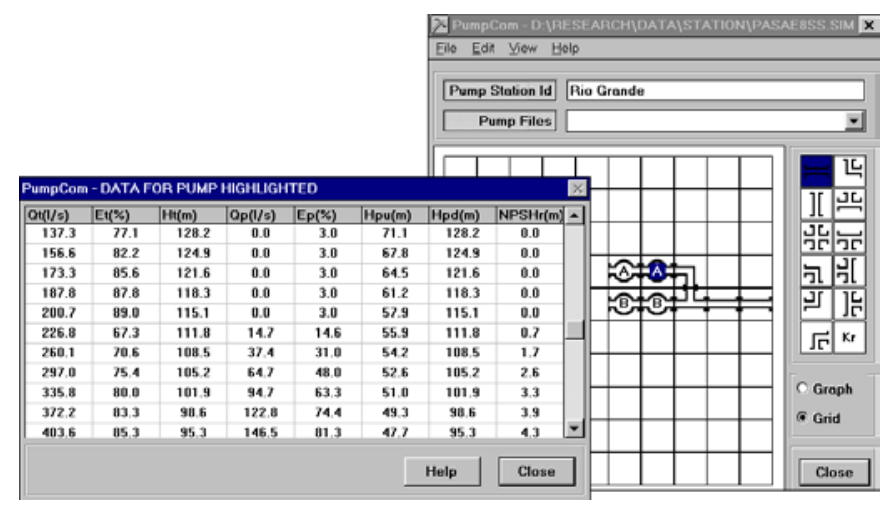

Figura 5. Dados da curva combinada da estação de bombeamento e da bomba A marcada no leiaute

e de potencial perigo de cavitação podem ser detectados e corrigidos antes que a estação de bombeamento seja instalada no campo.

Os dados da estação de bombeamento podem ser visualizados em gráfico ou impressos. Arquivos existentes podem ser editados da mesma forma como é feito para arquivos individuais de bombas, descrito anteriormente. O conjunto de dados da estação de bombeamento pode ser gravado em um arquivo para que possa ser utilizado depois por algum sistema de simulação de distribuição de água ou de irrigação, entre os quais o SPRINKMOD. Apesar de polinômios de grau até cinco poderem ser ajustados aos dados da estação de bombeamento, é pouco provável que o ajuste seja bom para o caso de bombas com capacidades diferentes sendo combinadas. Para superar essa dificuldade, o modelo de simulação maior, SPRINKMOD, utiliza "spline" cúbica para interpolar valores diretamente das tabelas de vazão-pressão; assim, qualquer tipo de curva combinada pode ser bem descrita nos processos de simulação de sistemas hidráulicos (Andrade, 1997; Andrade \& Allen, 1999).

O programa PUMPCOM pode ser operado sozinho, mas está também incorporado ao modelo de simulação maior, SPRINKMOD.

\section{Impressão de dados de curvas de bombas e de estações de bombeamento}

A tabela de dados de vazão versus pressão, eficiência e NPSHr para cada uma das bombas e para a estação de bombeamento pode ser enviada para ser impressa na impressora que estiver conectada ao computador. O mesmo conjunto de dados pode ser gravado em arquivo no formato delimitado por vírgula ou por aspas, o qual pode ser importado como texto em planilhas eletrônicas. Caixas de diálogo, ou o que estiver visível na tela do computador, pode ser capturado empregando-se a tecla, "Print Screen". O conteúdo do "clipboard" pode ser então colado dentro de um editor gráfico ou de texto e ser editado e impresso normalmente. Pressionando-se a tecla "Print Screen" enquanto a tecla "Alt" estiver pressionada, faz-se com que somente a janela ativa seja capturada para o "clipboard".

\section{EXEMPLO DE UTILILIZAÇÃO E VALIDAÇÃO DO MODELO}

Para que os cálculos executados no modelo pudessem ser verificados, combinaram-se as curvas de algumas bombas em série e em paralelo, utilizando-se o PUMPCOM (Figura 4) e também uma planilha eletrônica; inicialmente, duas bombas tipo "A" e duas tipo "B" foram combinadas em série e depois em paralelo (Figura 6).

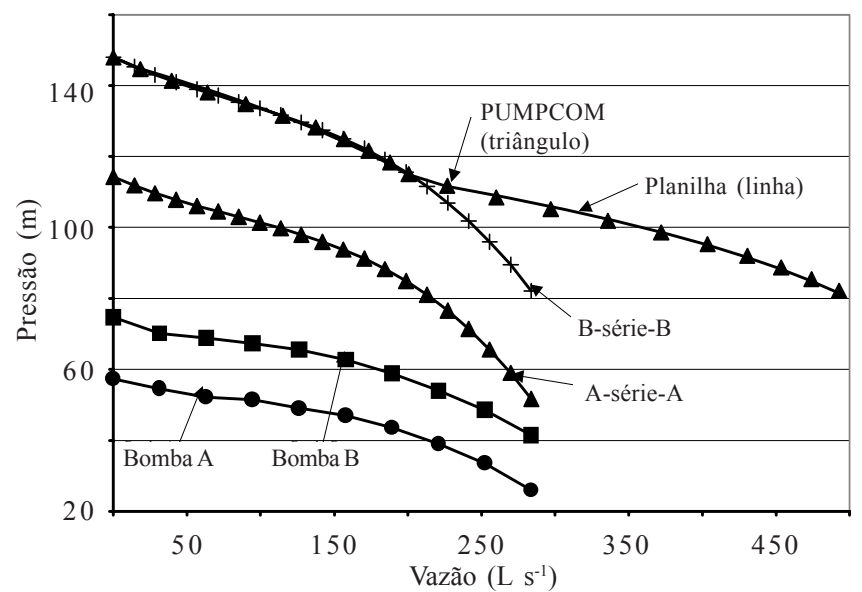

Figura 6. Vazão e pressão de bombas individuais e combinadas em série e depois em paralelo, como no leiaute da Figura 4, determinadas pelo PUMPCOM e calculadas empregando-se planilha eletrônica

Um polinômio de grau três foi ajustado aos dados de vazão versus pressão e versus eficiência, quando se utilizou a planilha eletrônica, enquanto o PUMPCOM emprega "spline" cúbica para interpolar os dados. Como a bomba "B" é maior que a bomba "A", a curva combinada coincide com a curva das bombas "B-série-B" na faixa de zero até $200 \mathrm{~L} \mathrm{~s}^{-1}$, significando que nessa faixa de vazão as duas bombas "A" não estão bombeando nenhuma água (Figura 5). Assumiu-se que, nesta faixa, as bombas "A" estão desligadas. Após $200 \mathrm{~L} \mathrm{~s}^{-1}$ as bombas "A" começam a bombear água e a curva combinada diverge da curva de "B-série-B". Os dados de vazão-pressão gerados pelo PUMPCOM se comparam muito bem com os dados da planilha eletrônica, o que confirma a acurácia do modelo (Figura 6).

A curva de eficiência para duas bombas idênticas, ligadas de forma similar, é a mesma; portanto, as curvas de vazãoeficiência para "A-série-A" e "B-série-B", são iguais às respectivas curvas individuais. A dificuldade surge quando "A-série-A" e "B-série-B" são combinadas em paralelo. Tal como para pressão, a curva combinada segue a curva "B-série-B" até $200 \mathrm{~L} \mathrm{~s}^{-1}$. Após este valor, a eficiência combinada entre as bombas "A-série-A" e "B-série-B" é estimada utilizando-se a Eq. 3. Como logo após $200 \mathrm{~L} \mathrm{~s}^{-1}$, as bombas "A" estão operando com uma vazão baixa (Figura 5) e, conseqüentemente, têm baixa eficiência (aproximadamente 14\%) e as bombas "B" estão em seu ponto de maior eficiência $(88 \%)$, a eficiência combinada cai para valores próximos de $65 \%$; esta eficiência sobe depois para $88 \%$, quando a estação de bombeamento está operando com $500 \mathrm{~L} \mathrm{~s}^{-1}$ (Figura 7).

A análise dos dados e gráficos permite verificar que, na faixa de zero a $200 \mathrm{~L} \mathrm{~s}^{-1}$, as bombas "A" devem permanecer desligadas; na faixa de 200 a $400 \mathrm{~L} \mathrm{~s}^{-1}$, a eficiência das bombas "A" será baixa e elas podem cavitar; portanto, para este exemplo, o ponto em que todas as bombas estariam operando adequadamente é o de $500 \mathrm{~L} \mathrm{~s}^{-1}$. 


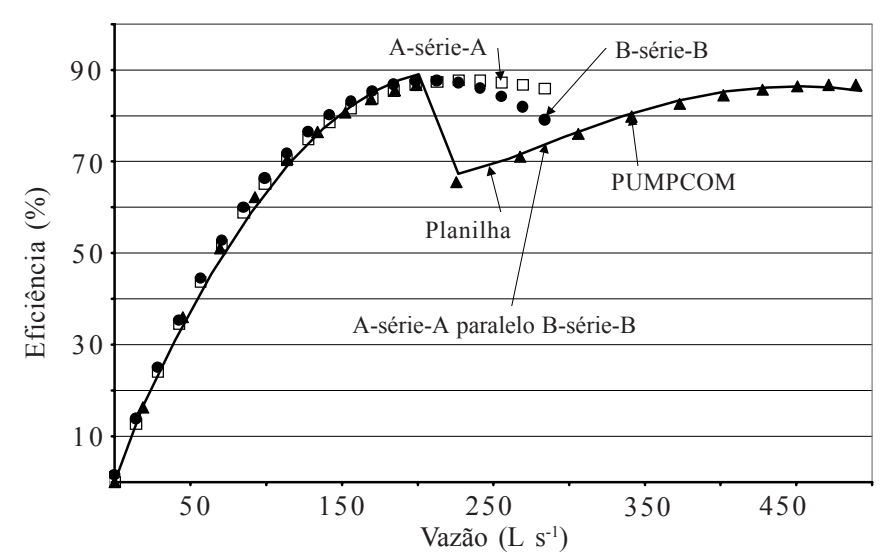

Figura 7. Vazão e eficiência para a estação de bombeamento e para uma das bombas da Figura 4, determinadas pelo PUMPCOM e empregando-se planilha eletrônica

Os valores de eficiência combinada simulados pelo PUMPCOM concordam bem com os valores calculados na planilha eletrônica (Figura 7). As pequenas diferenças entre valores simulados e calculados devem-se ao fato de o PUMPCOM empregar "spline" cúbica para interpolar entre dois pontos da curva, enquanto na planilha eletrônica um polinômio do terceiro grau foi utilizado para toda a faixa de vazão das bombas. Todavia, os dados de pressão combinada indicam um excelente ajuste (Figura 6).

Quando coeficientes de perda de carga localizada são incluídos na bomba "A" mais a montante, na bomba " $\mathrm{B}$ " mais a jusante e na interseção tipo "T" (Figura 4), uma curva diferente de vazão-pressão é obtida (Figura 8). A perda de carga aumenta com a vazão para um mesmo diâmetro de tubulação (Eq. 1), o que faz com que as curvas se afastem a medida em que a vazão aumenta (Figura 8). A concordância entre os dados de saída do modelo e os cálculos da planilha eletrônica é muito boa com e sem a inclusão das perdas de carga localizada, o que demonstra mais uma vez a sua acurácia.

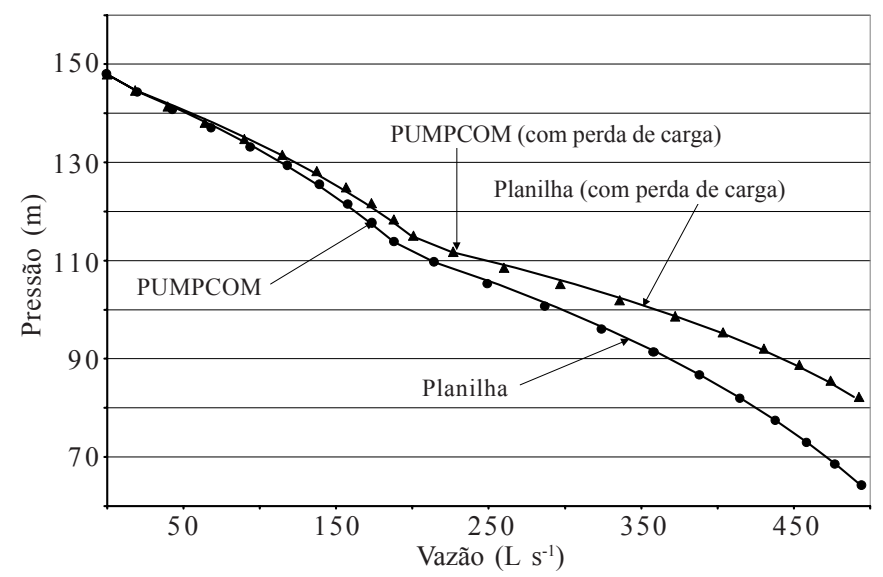

Figura 8. Vazão e pressão para a estação de bombeamento da Figura 4, com e sem perda de carga localizada, calculadas pelo PUMPCOM e empregando-se planilha eletrônica

\section{CONCLUSÕES}

1. A interface do modelo PUMPCOM simplifica a entrada de dados e a análise de resultados do processo de combinação de bombas em série e em paralelo.
2. O uso de polinômio de até quinto grau para descrever curvas individuais e de "spline" cúbica para interpolar dados entre pontos das curvas combinadas torna o modelo capaz de resolver os mais intricados sistemas de bombeamento.

3. Indicadores de desempenho para a estação de bombeamento e para cada uma das bombas que a compõem permitem uma análise detalhada do sistema para toda a sua faixa de operação.

4. A comparação entre os cálculos executados pelo PUMPCOM e empregando planilha eletrônica mostra que o modelo é acurado o suficiente para torná-lo de uso prático, mesmo considerando-se perdas de carga localizada no sistema.

\section{AGRADECIMENTO}

À Empresa Brasileira de Pesquisa Agropecuária - Embrapa e à "Utah Agricultural Experimental Station", dos Estados Unidos, cujo suporte financeiro foi fundamental na execução deste trabalho.

\section{LITERATURA CITADA}

Allen, R.G. Sprinkle irrigation design. BIE-607 Sprinkle irrigation design course notes. Logan: Utah State University, 1992. $272 \mathrm{p}$.

Andrade, C. de L.T. de Pressure and discharge distribution simulation in pressurized irrigation systems. Logan: Utah State University, 1997. 276p. PhD Thesis

Andrade, C. de L.T de; Allen R.G. SPRINKMOD - Pressure and discharge simulation model for pressurized irrigation systems. 1. Model development and description. Irrigation Science, Berlin, v.18, n.3, p.141-148, 1999.

Andrade, C. de L.T de; Allen R.G.; Wells R.D. SPRINKMOD - Pressure and discharge simulation model for pressurized irrigation systems. 3. Sensitivity to lateral hydraulic parameters and leakage. Irrigation Science, Berlin, v.18, n.3, p.157-161, 1999a.

Andrade C. de L.T de; Wells R.D; Allen R.G. SPRINKMOD - Pressure and discharge simulation model for pressurized irrigation systems. 2. Case study. Irrigation Science, Berlin, v.18, n.3, p.149-156, 1999 b.

Flammer, G.H.; Jeppson R.W.; Keedy H.F. Fundamental principles and applications of fluid mechanics. Logan: Utah State University, Department of Civil and Environmental Engineering, 1986. 376p.

Gessler, J. Analysis of pipe networks. In: Chaudhry, M.H.; Yevjevich, V. (eds.). Closed-conduit flow. Chelsea: Water Resources Publication, 1981. Cap.4, p.61-100.

Keller, J.; Bliesner, R.D. Sprinkle and trickle irrigation. New York: van Nostrand Reinhold, 1990. 652p.

Kincaid; D.R.; Cheney, E.W. Numerical analysis - Mathematics of scientific computing. Pacific Grove: Brooks/Cole, 1991. 690p.

Krivchenko, G. Hydraulic machines: Turbines and pumps. Boca Raton: Lewis Publishers, 1994. 273p.

Manson, J.C. BASIC matrix methods. London: Butterworths, 1987. 160p. 
Mathews, J.H. Numerical methods for computer science, engineering and mathematics. Cliffs: Prentice Hall, 1987.378p.

Molina, E. Simulation model to predict operating pressures and flow rates for a sprinkler system in operation. Logan: Utah State University, 1991. 129p. Master's Dissertation

Ormsbee, L.E.; Walski T.M. Identifying efficient pump combinations. Journal of Water Works Association, Denver, v.8, n.1, p.31-34, 1989.

Ramalan, A.A. Management strategies for gravity sprinkle irrigation system. Logan: Utah State Univiversity, 1988. 198p. PhD Thesis
Tarquin, A.J.; Dowdy, J. Optimal pump operation in water distribution. Journal of Hydraulic Engineering, New York, v.115, n.2, p.158-168, 1989.

Tullis, J.P. Hydraulics of pipelines: Pumps, valves, cavitation, transients. New York: J. Wiley. 1989. 266p.

Wells, R.D.; Busch, J. Optimal operating rules for a multiple unit pumping plant. Comunicação pessoal. 1981. 5p.

Wheeler, L.A. A model for predicting the performance of electrical irrigation pumping plants. Logan: Utah State University. 1993. 142p. Master's Dissertation 